\title{
Joint Optimization of Carrier Sensing Threshold and Transmission Rate in Wireless Ad Hoc Networks
}

\author{
(Invited Paper)
}

\author{
Yongping Zhang, Bo Li, Mao Yang, Zhongjiang Yan, and Xiaoya Zuo \\ School of Electronics and Information \\ Northwestern Polytechnical University, Xi'an, China \\ Email: zhyp2010@mail.nwpu.edu.cn \\ \{libo.npu, yangmao, zhjyan, zuoxy\}@nwpu.edu.cn
}

\begin{abstract}
The ever-increasing demands of wireless traffic call for continuously growing the wireless network capacity or throughput. Carrier sensing threshold adaptation and transmission rate adaptation, two efficient mechanisms improving the capacity of ad hoc network, have attracted much attention in recent years. However, simply adopting either mechanism can hardly meet the expectation that optimizing the network capacity. In this paper, we investigate the joint optimization of carrier sensing threshold and transmission rate to improve the network capacity or throughput. The area capacity (throughput), which is defined as the network capacity (throughput) per unit area, is theoretically analyzed. Meanwhile, the relationship between carrier sensing threshold and transmission rate is theoretically revealed. Furthermore, the optimal area capacity is obtained by jointly optimizing the carrier sensing threshold and the transmission rate. Simulation results validate our analysis and derived results, and show that the throughput is significantly improved by the joint optimization.
\end{abstract}

Keywords-Carrier Sensing Threshold; Transmission Rate; Area Throughput; Area Capacity.

\section{INTRODUCTION}

With the proliferation of mobile devices and the rapid development of multimedia applications, the high-density deployment of wireless networks becomes a growing trend. How to improve the capacity, or the aggregate throughput, to fulfill the ever-increasing bandwidth requirements becomes a hot topic. As surveyed in [1], the promising approaches can be divided into two categories, the temporal approach, mainly refers to the contention window adaptation, and the spatial approach or spatial reuse, including the carrier sensing threshold adaptation, the transmission rate adaptation, the transmission power control and the use of directional antenna. In recent years, there are many efforts focusing on the carrier sensing threshold adaptation [2]-[7] and transmission rate adaptation [8]-[10].

Carrier sensing threshold adaptation plays an important role in the interference management and throughput enhancement in the carrier sensing multiple access (CSMA) based wireless networks. Cho et al. [3] use the carrier sensing threshold adaptation to control the density of secondary users and reduce the resulting interference level for primary system in cognitive wireless networks. Kaynia et al. [4] investigate the optimal carrier sensing threshold in terms of the outage probability. Zhang et al. [5] classify the neighbouring areas of an ongoing transmission into three areas: hidden area, exposed area and overlapped area, and propose a coordinated dynamic physical carrier sense (CDPCS) scheme based on local optimization to adjust the carrier sensing threshold of the neighbouring nodes by the exchange of simplified request-to-send (SRTS) and simplified clear-to-send (SCTS). Further, Zhang et al. [6] introduce a throughput penalty model to derive the optimal carrier sensing threshold and propose an iterative algorithm to adjust the carrier sensing threshold through statistical channel state. Yang et al. [7] extend both Bianchi's and Kumar's models to investigate the impact of transmit power and carrier sense threshold on network capacity, and find that high throughput can be achieved with the condition that the carrier sensing reservation zone of the sender covers the interference range of the intended receiver. This valuable finding inspires our research. These studies show that the interference caused by simultaneous transmissions [3], [4] or hidden and exposed terminals [5]-[7] can be mitigated effectively by tuning the carrier sensing threshold. However, the interference tolerance level of the communication link is determined by the required signal-to-interference-noise ratio (SINR) corresponding to the given transmission rate. Therefore, simply adapting the carrier sensing threshold can hardly achieve the maximum network throughput.

Selecting appropriate transmission rates according to channel condition can improve the bandwidth utilization efficiency. Cardoso et al. [8] propose a standards-compliant rate adaptation algorithm by measuring the contention level to identify the packet losses due to collision for dense IEEE 802.11 networks. Huang et al. [9] propose a transmission rate adaptation for colliding links (TRACK) protocol to improve the throughput by enabling the concurrent transmissions of exposed terminals with appropriate transmission rates. Blaich et al. [10] hypothesize that transmission rate adaptation should be justified with regard to the global throughput of the wireless network rather than the individual nodes. A concept of rate zone is introduced to justify the rate adaptation, and the experimental, simulation, and real-world results support the hypothesis. Selecting a best transmission rate can take advantage of the received SINR [8], [9], however, the interference caused by potential transmissions degrades the link reliability, and the optimization for individual link or local area may bring adverse impact on the network throughput [10]. Therefore, the interference induced by simultaneous transmissions in the whole network should be controlled to select an appropriate transmission rate.

Joint adaptation of carrier sensing threshold and transmis- 
sion rate is considered in [11]-[13]. Yang et al. [11] introduce the concept of spatial backoff and adjust the occupied space of transmissions to improve the aggregate throughput. A dynamic algorithm based on joint control of carrier sensing threshold and transmission rate is proposed to realize the spatial backoff. Subsequent research in [12] takes the small-scale multipath fading channel into account. Although the spatial backoff algorithms achieve competitive network throughput, the factors impacting on the optimal carrier sensing threshold and transmission rate are to be resolved. Kim et al. [13] develop an analytical model to investigate the network capacity with joint carrier sensing threshold and transmission rate adaptation, and show that the optimal carrier sensing range can approximated by the sum of the link distance and the interference range of the receiver with a given transmission rate. Further, the contention window size is optimized to achieve the maximum throughput, and a distributed link adaptation and contention control scheme (LACC) is proposed. However, the optimal values of carrier sensing threshold, transmission rate and contention window size cannot be obtained theoretically. The effectiveness of joint optimization of carrier sensing threshold adaptation and transmission rate adaptation in existing research motivates us to analyze the optimal network capacity or throughput, and derive the theoretical results of optimal carrier sensing threshold and transmission rate.

In this paper, we aim to obtain the joint optimization of carrier sensing threshold and transmission rate to improve the network capacity or throughput. The concept of area capacity is introduced, and the relationship between the carrier sensing threshold and transmission rate is theoretically revealed. Then, we derive the expression of the area capacity based on the theoretical Shannon capacity and obtain the optimal carrier sensing threshold, optimal transmission rate and corresponding area capacity. The results can be extended to the analysis of area throughput with the practical discrete transmission rates readily. Simulation results validate our analysis, and show that the throughput is significantly improved by the joint optimization.

The contributions of this paper can be summarized as follows:

- We derive the expression of the area capacity based on the theoretical Shannon capacity, and obtain the optimal carrier sensing threshold, optimal transmission rate and corresponding area capacity.

- Based on the analysis of area capacity, the expression of the area throughput is also derived, and the corresponding optimal values of the carrier sensing threshold and transmission rate can be obtained readily.

- Simulation results validate the optimal values of carrier sensing threshold and transmission rate, and show that the throughput is improved by the joint optimization.

The remainder of the paper is organized as follows. In Section II, we describe the system model and introduce the definitions of the related parameters. In Section III and Section IV, we present the analysis and optimization of the area capacity and area throughput, respectively. In Section V, we validate the analysis through simulation. Finally, we conclude this paper in Section VI.

\section{SyStem MODEL}

We consider a wireless network in which the senders are randomly located on an area of $A$ with the Poisson point process (PPP) distribution. The transmit power of $P_{t}$ is uniform and constant, and the distance between any sender and its receiver is fixed as $d$. For the propagation model, a path loss model is considered while both the short-term and long-term fading are ignored. Therefore, the received power of $P_{r}$ can be modeled as

$$
P_{r}=\frac{K P_{t}}{d^{\alpha}},
$$

where $\alpha$ is the path loss exponent (PLE) and is normally in the range between 2 and 4 . Other parameters of propagation model are termed as an uniform $K$. Without loss of generality, we set $K=1$.

For a typical communication link with a sender $S$ and its intended receiver $R$, the reception at $R$ is considered successful at a certain transmission rate if the received SINR exceeds a predefined SINR threshold, i.e.,

$$
P_{r} /\left(N_{0}+I\right) \geq \gamma_{t h},
$$

where $\gamma_{t h}$ denotes the SINR threshold, $N_{0}$ and $I$ denote the noise power and interference power respectively.

According to the propagation and interference models, the definitions of interference range, carrier sensing range and carrier sensing reservation zone are given as follows.

Definition 1 (Interference Range, $d_{i n}$ ): Interference range is defined as the range within which once any node sends data, the reception of $R$ will be interfered. In this case, the resulting received SINR is below the SINR threshold, $\gamma_{t h}$. It can be derived as

$$
d_{i n}=\gamma_{t h}^{\frac{1}{\alpha}} \cdot d,
$$

in which the noise power of $N_{0}$ is ignored.

Definition 2 (Carrier Sensing Range, $d_{c s}$ ): Carrier sensing range is defined as the range within which once any node sends data, it will be detected by the potential sender of $S$. In this case, the sensing energy on the channel exceeds the predefined carrier sensing threshold, $C S_{t h}$. It can be derived as

$$
d_{c s}=\left(\frac{P_{t}}{C S_{t h}}\right)^{\frac{1}{\alpha}} .
$$

Definition 3 (Carrier Sensing Reservation Zone, $d_{r z}$ ): Carrier sensing reservation zone is defined as the region within which once the sender of $S$ sends data, it will be detected by any other node. It is exactly equal to the carrier sensing range with the condition of a uniform transmit power and carrier sensing threshold, i.e.,

$$
d_{r z} \equiv d_{c s} .
$$

For convenience, a margin, $\theta$, is introduced to characterize the difference between the received signal strength and carrier sensing threshold as

$$
\theta=\frac{P_{r}}{C S_{t h}} .
$$

Thus, the carrier sensing range can be expressed by

$$
d_{c s}=\theta^{\frac{1}{\alpha}} \cdot d .
$$




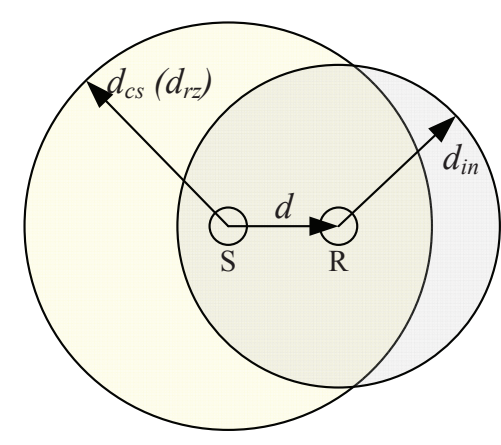

Fig. 1. Parameters in basic communication link. $d$ denotes the distance between sender $S$ and receiver $R . d_{c s}, d_{r z}$ and $d_{i n}$ denote the carrier sensing range, size of carrier sensing reservation zone and interference range respectively.

Note that any carrier sensing threshold can be indicated by the margin of $\theta$ with a fixed sender-receiver distance of $d$. The relationship of $d, d_{i n}, d_{c s}$ and $d_{r z}$ is shown in the Fig. 1.

\section{AREA CAPACITY ANALYSIS AND OPTIMIZATION}

\section{A. Definition of Area Capacity}

The spatial reuse can be improved by the carrier sensing threshold adaptation or (and) transmission rate adaptation. The carrier sensing threshold adaptation controls the interference level in the wireless network, while the transmission rate adaptation can take advantage of the received SINR. Thus, it is necessary to reveal the relationship of these two mechanisms and further jointly optimize them to improve the network capacity. In order to characterize the effect of joint optimization, a concept of area capacity is introduced as follows.

Definition 4 (Area Capacity): Area capacity is defined as the ratio of the total channel capacity of successful links to the network coverage area. It can be expressed by

$$
C_{a} \triangleq \frac{\sum_{i=1}^{n} C_{i}}{A}
$$

where $C_{i}$ and $A$ denote the channel capacity of link $i$ and the network coverage area respectively.

Note that a uniform transmit power and carrier sensing threshold is used in the network, the carrier sensing range and corresponding transmission rate which is select according to the maximum tolerable interference range are identical for all nodes, i.e., $C_{i}=C$ for $i=1,2, \cdots, n$. Therefore, the area capacity can be converted to be

$$
C_{a}=\lambda C,
$$

where $\lambda=n / A$ denotes the link density of the network.

\section{B. Area Capacity Analysis}

The link density of the network can be obtained by the following theorem.

Theorem 1: In the homogeneous wireless networks, the maximum link density of simultaneous transmissions is $\frac{1}{e \pi d^{2}} \frac{1}{\theta^{2 / \alpha}}$ under the condition that $Z \ll A$, where $Z$ denotes the area of carrier sensing reservation zone.
Proof: For any transmitting node, its transmission will be collided when another transmitting node locates inside its carrier sensing reservation zone. Thus, its collision probability is $p=Z / A$. If $N$ nodes start transmitting simultaneously, the success probability of one link is $p_{s}=(1-p)^{N-1}$, and then the average number of successful links can be expressed by

$$
N_{s}=N \cdot p_{s}=N(1-p)^{N-1}=N\left(1-\frac{Z}{A}\right)^{N-1} .
$$

Take natural logarithm on both side of the Eq. (10), then we have

$$
\ln N_{s}=\ln N+(N-1) \ln \left(1-\frac{Z}{A}\right) .
$$

Take the derivation of $N$ on the right side of the Eq. (11) and let the derivation be zero, then we can obtain the maximum average number of successful links expressed by

$$
N_{s}=\frac{A}{Z}\left(1-\frac{Z}{A}\right)^{\left(\frac{A}{Z}-1\right)},
$$

which is attained at $N \approx \frac{A}{Z}$ under the condition that $Z \ll A$. Therefore, we obtain the link density of simultaneous transmissions expressed by

$$
\lambda=\frac{N_{s}}{A}=\frac{1}{\left(1+\frac{Z}{A-Z}\right)^{\frac{A-Z}{Z}} \cdot Z} \approx \frac{1}{e Z} .
$$

It suggests that the link density can be maximized as $1 /(e Z)$ with the number of contending nodes optimized as $A / Z$ by an appropriate medium access control (MAC) protocol. Finally, the area of carrier sensing reservation zone can be calculated by $Z=\pi d_{r z}^{2}$, substituting the expression of $d_{r z}$, and then we can obtain the link density expressed by

$$
\lambda=\frac{1}{e \pi d^{2}} \frac{1}{\theta^{2 / \alpha}} .
$$

For any given transmission rate, the close-to-optimal throughput can be achieved by an appropriate carrier sensing threshold eliminating the hidden terminals [7], [13]. Thus, the transmission rate for the communication link is determined by the maximum tolerable interference range, which is depicted in Fig. 2. In order to eliminate the hidden terminals around $R$, the interference range of $R$ should be covered by the carrier sensing reservation zone of $S$, then we have

$$
d_{r z}=d+d_{i n} .
$$

Recalling the definitions of the related parameters in Section II, we can obtain that

$$
\theta^{1 / \alpha}=1+\gamma_{t h}^{1 / \alpha}
$$

A given carrier sensing threshold corresponding to the margin of $\theta$ determines the SINR threshold of $\gamma_{t h}$. According to Shannon's formula, the channel capacity, as the upper bound of transmission rate, is expressed by $C=\log \left(1+\gamma_{t h}\right)$. Note that $\log (\cdot)$ in this paper is to base 2 . Therefore, the area capacity can be obtained as

$$
C_{a}=\frac{1}{e \pi d^{2}} \frac{\log \left(1+\gamma_{t h}\right)}{\left(1+\gamma_{t h^{\frac{1}{\alpha}}}\right)^{2}}
$$




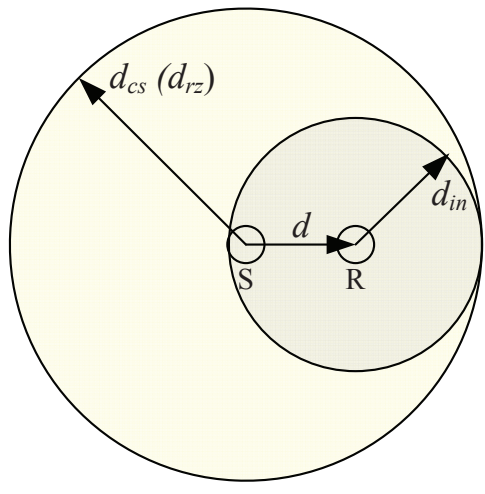

Fig. 2. The relationship between the transmission rate and carrier sensing threshold. The interference range of $R$ should be covered by the carrier sensing reservation zone of $S$, then hidden terminals around $R$ are eliminated.

\section{Area Capacity Optimization}

Optimizing the area capacity in (17) is equivalent to maximizing the function

$$
f\left(\gamma_{t h}\right)=\frac{\log \left(1+\gamma_{t h}\right)}{\left(1+\gamma_{t h}{ }^{\frac{1}{\alpha}}\right)^{2}} .
$$

Taking the derivation of $\gamma_{t h}$ on $f\left(\gamma_{t h}\right)$, then we have

$$
f^{\prime}\left(\gamma_{t h}\right)=\frac{1-\frac{\gamma_{t h}{ }^{\frac{1}{\alpha}}}{1+\gamma_{t h}^{\frac{1}{\alpha}}} \cdot \frac{1+\gamma_{t h}}{\gamma_{t h}} \cdot \frac{2}{\alpha} \ln \left(1+\gamma_{t h}\right)}{\ln 2 \cdot\left(1+\gamma_{t h}\right)\left(1+\gamma_{t h}{ }^{\frac{1}{\alpha}}\right)^{2}} .
$$

Note that the PLE of $\alpha$ is usually not too large, If the received SINR threshold $\gamma_{t h}$ is large, then the expression can be simplified to be

$$
f^{\prime}\left(\gamma_{t h}\right) \approx \frac{1-\frac{2}{\alpha} \ln \gamma_{t h}}{\ln 2 \cdot\left(1+\gamma_{t h}\right)\left(1+\gamma_{t h}^{\frac{1}{\alpha}}\right)^{2}} .
$$

Let the derivation be zero, it is easy to obtain the approximate expression for the optimal SINR threshold as

$$
\gamma_{t h}^{*}=e^{\frac{\alpha}{2}} \text {. }
$$

The corresponding optimal size of carrier sensing threshold and optimal area capacity are derived as

$$
\theta^{*}=(\sqrt{e}+1)^{\alpha},
$$

and

$$
C_{a}^{*} \approx \frac{\alpha}{(2 \pi \ln 2)(\sqrt{e}+1)^{2} d^{2}}
$$

respectively.

From Eq. (21) to Eq. (23), it is observed that the optimal transmission rate, carrier sensing threshold and area capacity increase with the increasing of PLE.

\section{AREA Throughrut ANALYSIS}

Practically, the available transmission rates in the wireless network are limited and discrete, and different transmission rates require different SINR thresholds. The reception is considered successful at a certain transmission rate if the received SINR exceeds the corresponding SINR threshold. Assume $m$ discrete transmission rates are available as $\{R(k), k=$ $1,2, \cdots, m\}$, and the received SINR corresponding to the transmission rate of $R(k)$ is in the range of $\left[\gamma_{t h}(k)\right), \gamma_{t h}(k+$ $1))$. Therefore, the relationship between the transmission rate and received SINR is given by $R(\gamma)=R(k)$, where $\gamma$ denotes the received SINR and $\gamma \in\left[\gamma_{t h}(k), \gamma_{t h}(k+1)\right)$. In order to characterize the effect of joint optimization of the carrier sensing threshold and transmission rate on the network throughput, a concept of area throughput is introduced as follows.

Definition 5 (Area Throughput): Area throughput is defined as the ratio of the total transmission rate of successful links to the network coverage area. It can be expressed by

$$
S_{a} \triangleq \frac{\sum_{i=1}^{n} R_{i}}{A}
$$

where $R_{i}$ denotes the transmission rate of link $i$.

Similar to the area capacity, all nodes select the same transmission rate based on the uniform transmit power and carrier sensing threshold, i.e., $R_{i}=R$ for $i=1,2, \cdots, n$. Therefore, the area throughput can be converted to be

$$
S_{a}=\lambda R .
$$

The transmission rate is determined by the received SINR of $\gamma$, recalling the link density of the wireless network in Theorem 1, and then we have

$$
S_{a}=\frac{R(\gamma)}{e \pi d^{2} \theta^{2 / \alpha}} .
$$

The received SINR will fall in a certain interval of $\left.\left[\gamma_{t h}(k)\right), \gamma_{t h}(k+1)\right)$, and the corresponding transmission rate becomes $R(\gamma)=R(k)$. Recalling the relationship between the carrier sensing threshold and transmission rate in (16), the minimum of $\theta$ is expressed by

$$
\theta_{\min }(k)=\left[1+\gamma_{t h}{ }^{1 / \alpha}(k)\right]^{\alpha} .
$$

Therefore, the area throughput can be derived as

$$
S_{a}=\frac{1}{e \pi d^{2}} \frac{R(k)}{\left[1+\gamma_{t h^{\frac{1}{\alpha}}}(k)\right]^{2}} .
$$

The optimal transmission rate can be obtained readily according to (28), and then the corresponding optimal carrier sensing threshold can be derived by (27).

Taking an example of IEEE 802.11a, the required SINR threshold to support the corresponding transmission rate [14] is shown in Table I. The area throughput is normalized by the fixed factor of $\frac{1}{e \pi d^{2}}$, i.e.,

$$
\hat{S}_{a}=\frac{R(k)}{\left[1+\gamma_{t h^{\frac{1}{\alpha}}}(k)\right]^{2}} .
$$

The normalized area throughput of $\hat{S}_{a}$ for some typical PLEs are also shown in the table.

Note that $\hat{S}_{a}(\cdot)$ denotes the normalized area throughput with the corresponding PLE. To maximize the area throughput, the optimal transmission rate can be found out in Table I. Then the optimal carrier sensing threshold can be derived by (27). The optimal transmission rate, carrier sensing threshold and normalized area throughput for $\alpha=2,3,4$ are summarized 


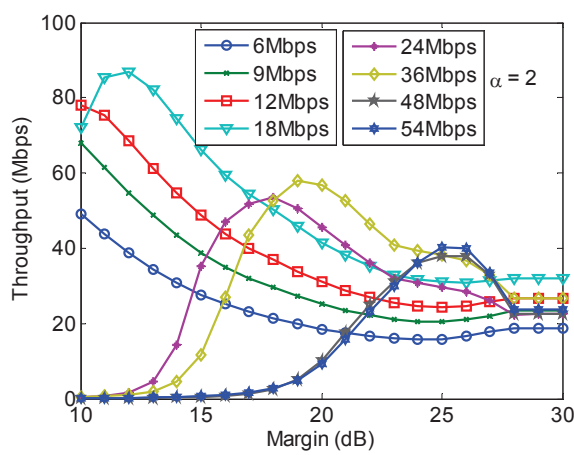

(a) $\alpha=2$

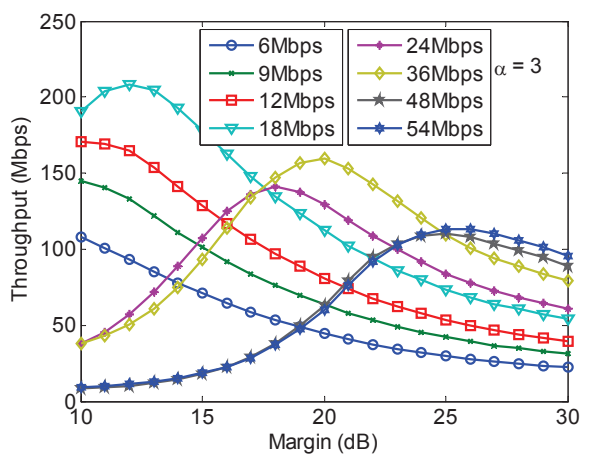

(b) $\alpha=3$

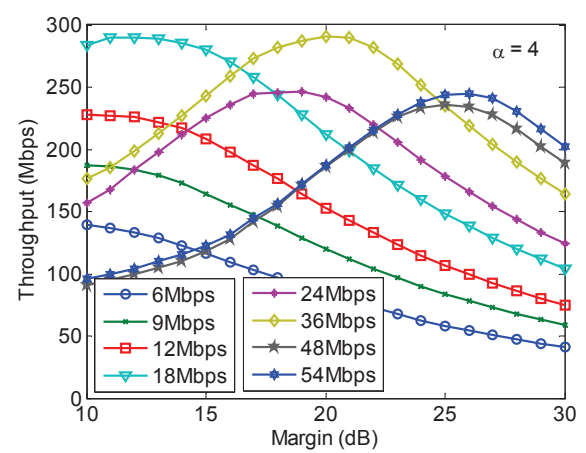

(c) $\alpha=4$

Fig. 3. Throughput with the different carrier sensing thresholds for each fixed transmission rate. The joint optimizations of carrier sensing threshold and transmission rate are (12 dB, $18 \mathrm{Mbps}),(12 \mathrm{~dB}, 18 \mathrm{Mbps})$ and $(20 \mathrm{~dB}, 36 \mathrm{Mbps})$ for $\alpha=2,3,4$.

TABLE I. TRANSMISSION RATE, SINR THRESHOLD, AND NORMALIZED NETWORK THROUGHPUT

\begin{tabular}{l|c|c|c|c}
\hline$k(R(k))$ & $\gamma_{t h}(k)$ & $\hat{S}_{a}(2)$ & $\hat{S}_{a}(3)$ & $\hat{S}_{a}(4)$ \\
\hline $1(6 \mathrm{Mbps})$ & $6.02 \mathrm{~dB}$ & 0.67 & 0.90 & 1.03 \\
\hline $2(9 \mathrm{Mbps})$ & $7.78 \mathrm{~dB}$ & 0.76 & 1.13 & 1.37 \\
\hline $3(12 \mathrm{Mbps})$ & $9.03 \mathrm{~dB}$ & 0.82 & 1.33 & 1.67 \\
\hline $4(18 \mathrm{Mbps})$ & $10.79 \mathrm{~dB}$ & $\mathbf{0 . 9 0}$ & $\mathbf{1 . 6 6}$ & 2.20 \\
\hline $5(24 \mathrm{Mbps})$ & $17.04 \mathrm{~dB}$ & 0.36 & 1.09 & 1.78 \\
\hline $6(36 \mathrm{Mbps})$ & $18.80 \mathrm{~dB}$ & 0.38 & 1.31 & $\mathbf{2 . 3 1}$ \\
\hline $7(48 \mathrm{Mbps})$ & $24.05 \mathrm{~dB}$ & 0.17 & 0.90 & 1.93 \\
\hline $8(54 \mathrm{Mbps})$ & $24.56 \mathrm{~dB}$ & 0.17 & 0.94 & 2.07 \\
\hline
\end{tabular}

in Table II. The trend of higher optimal transmission rate and carrier sensing threshold for higher PLE is consistent with the analytical result based on the area capacity.

TABLE II. OPTIMAL TRANSMISSION RATE, CARRIER SENSING THRESHOLD AND NORMALIZED AREA THROUGHPUT

\begin{tabular}{c|c|c|c|c}
\hline & $R^{\text {opt }}$ & $\gamma_{t h}$ & $\theta^{\text {opt }}$ & $\hat{S_{a}}$ \\
\hline$\alpha=2$ & $18 \mathrm{Mbps}$ & $10.79 \mathrm{~dB}$ & $12.99 \mathrm{~dB}$ & 0.90 \\
\hline$\alpha=3$ & $18 \mathrm{Mbps}$ & $10.79 \mathrm{~dB}$ & $15.51 \mathrm{~dB}$ & 1.66 \\
\hline$\alpha=4$ & $36 \mathrm{Mbps}$ & $18.80 \mathrm{~dB}$ & $23.87 \mathrm{~dB}$ & 2.31 \\
\hline
\end{tabular}

\section{RESUlts AND DisCUSSIONS}

In the simulations, the classical distributed coordination function (DCF) mechanism adopted by IEEE 802.11 standard is used for channel access. On the one hand, for each available transmission rate, the carrier sensing threshold is varied by changing the margin of $\theta$ from $10 d B$ to $30 \mathrm{~dB}$, and then the aggregate throughput is investigated to validate the analysis. On the other hand, an ideal SINR-based rate adaptation scheme, in which the accurate channel SINR is assumed to be always available, is implemented for performance comparison. The throughput of ideal rate adaptation with optimal carrier sensing threshold (opt. CS \& ideal RA) and ideal rate adaptation with fixed carrier sensing threshold (fixed CS \& ideal RA) are investigated in the simulations. The simulation parameters are based on IEEE 802.11a, and the supported transmission rates and the corresponding SINR thresholds are listed in Table I. Other settings in the simulations are shown in Table III. In addition, only basic access mechanism with DATA and ACK (acknowledgement) is considered.

The coverage area of the considered network is a square of $400 m \times 400 m$ in the simulations. The locations of the senders

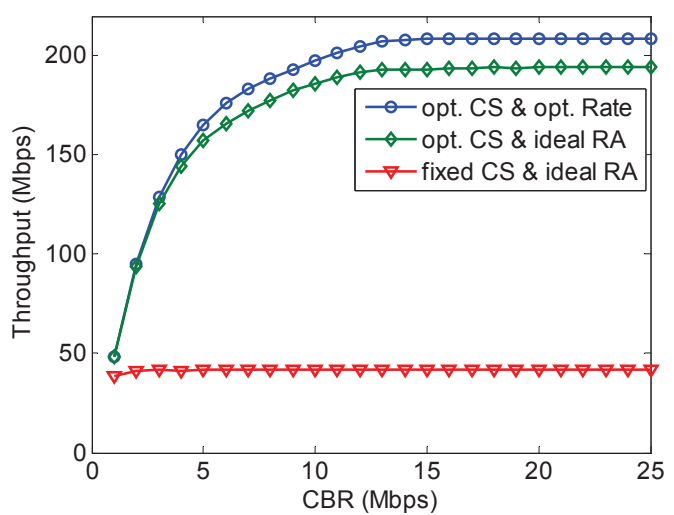

Fig. 4. Throughput with the increasing traffic rate $(\alpha=3)$. The throughput is improved by the joint optimization of the carrier sensing threshold and transmission rate.

are distributed with PPP and the average number of senders is 50. The intended receiver is randomly located on the circle with a fixed radius of $20 \mathrm{~m}$ around the sender. The simulation time is 30 seconds and the results are calculated by an average of 10 runs with different random topologies. The simulations are conducted by Network Simulator $2(n s-2)$ with version 2.35 .

TABLE III. SimUlation SETTINGS

\begin{tabular}{c|c|c}
\hline Symbol & Description & Value \\
\hline$P_{t}$ & transmit power & $0.01 \mathrm{~W}$ \\
$d$ & link distance & $20 \mathrm{~m}$ \\
$\varepsilon$ & fixed carrier sensing threshold & $-82 \mathrm{dBm}$ \\
$L$ & frame length & $1400 \mathrm{Byte}$ \\
\hline
\end{tabular}

Fig. 3 shows the network throughput with different carrier sensing threshold (converted to the margin, $\theta$ ) for each fixed transmission rate. It is obviously that, the joint optimizations of carrier sensing threshold and transmission rate are $(12 \mathrm{~dB}$, $18 \mathrm{Mbps}),(12 \mathrm{~dB}, 18 \mathrm{Mbps})$ and $(20 \mathrm{~dB}, 36 \mathrm{Mbps})$ for $\alpha=2,3,4$. Compared with the analytical results in Table II, the optimal transmission rates are exactly the same as the analytical results, and the optimal carrier sensing threshold have the same trend with the analytical results.

Fig. 4 shows that the throughput is improved by the joint optimization of the carrier sensing threshold and transmission 
rate. It is interesting that although the ideal rate adaptation can select the best transmission rate in real time according to the channel condition, the achieved throughput of ideal rate adaptation with the optimal carrier sensing threshold is not optimal. The reason is that, the ideal rate adaptation is optimized locally, and the interference caused by simultaneous transmissions in the whole network degrades the link reliability. In addition, a throughput gain about four times with PLE of 3 compared with the throughput of ideal rate adaptation with the fixed carrier sensing threshold $(-82 \mathrm{dBm})$ can be obtained.

\section{CONCLUSION AND FUture WORK}

In this paper, we investigate the joint optimization of carrier sensing threshold and transmission rate to improve the network capacity or throughput of wireless ad hoc networks. The relationship between the carrier sensing threshold and transmission rate is theoretically revealed, then the expression of the area capacity based on the theoretical Shannon capacity is derived and the optimal carrier sensing threshold, optimal transmission rate and corresponding area capacity is obtained. The results can be extended to the analysis of area throughput with the practical discrete transmission rates readily. Simulation results validate the optimal values of carrier sensing threshold and transmission rate, and show that the throughput is improved by the joint optimization. Further, a throughput gain about four times with PLE of 3 compared with the throughput of ideal rate adaptation with the fixed carrier sensing threshold (-82 $\mathrm{dBm}$ ) can be obtained. In the future, a practical MAC protocol based on the joint optimization of carrier sensing threshold and transmission rate will be investigated.

\section{ACKNOWLEDGMENT}

This work was supported in part by the National Natural Science Foundations of CHINA (Grant No. 61271279, and 61201157), the National 863 plans project (Grant No. 2014AA01A707, and 2015AA011307), the National Science and Technology Major Project (Grant No. 2015ZX03002006), and the Fundamental Research Funds for the Central Universities (Grant No. 3102015ZY038, 3102015ZY039).

\section{REFERENCES}

[1] B. Alawieh, Y. Zhang, C. Assi, and H. Mouftah, "Improving spatial reuse in multihop wireless networks-a survey," Communications Surveys \& Tutorials, IEEE, vol. 11, no. 3, pp. 71-91, 2009.

[2] C. Thorpe and L. Murphy, "A survey of adaptive carrier sensing mechanisms for ieee 802.11 wireless networks," Communications Surveys \& Tutorials, IEEE, vol. 16, no. 3, pp. 1266-1293, 2014.

[3] B. Cho, K. Koufos, and R. Jantti, "Interference control in cognitive wireless networks by tuning the carrier sensing threshold," in Cognitive Radio Oriented Wireless Networks (CROWNCOM), 2013 8th International Conference on. IEEE, 2013, pp. 282-287.

[4] M. Kaynia, N. Jindal, and G. E. Oien, "Improving the performance of wireless ad hoc networks through mac layer design," Wireless Communications, IEEE Transactions on, vol. 10, no. 1, pp. 240-252, 2011.

[5] X. Zhang, G. Qiu, Z. Dai, and D. K. Sung, "Coordinated dynamic physical carrier sensing based on local optimization in wireless ad hoc networks," in Wireless Communications and Networking Conference (WCNC), 2013 IEEE. IEEE, 2013, pp. 398-403.

[6] X. Zhang, H. Zhu, and G. Qiu, "Optimal physical carrier sensing to defend against exposed terminal problem in wireless ad hoc networks," in Computer Communication and Networks (ICCCN), 2014 23rd International Conference on. IEEE, 2014, pp. 1-6.

[7] Y. Yang, J. C. Hou, and L.-C. Kung, "Modeling the effect of transmit power and physical carrier sense in multi-hop wireless networks," in INFOCOM 2007. 26th IEEE International Conference on Computer Communications. IEEE. IEEE, 2007, pp. 2331-2335.

[8] K. V. Cardoso and J. F. de Rezende, "Increasing throughput in dense 802.11 networks by automatic rate adaptation improvement," Wireless Networks, vol. 18, no. 1, pp. 95-112, 2012.

[9] J. Huang, G. Xing, and G. Zhou, "Unleashing exposed terminals in enterprise wlans: A rate adaptation approach," in INFOCOM, 2014 Proceedings IEEE. IEEE, 2014, pp. 2481-2489.

[10] A. Blaich, S. Liu, and A. Striegel, "Re-thinking 802.11 rate selection in the face of non-altruistic behavior," in Computer Communications and Networks (ICCCN), 2013 22nd International Conference on. IEEE, 2013, pp. 1-6.

[11] X. Yang and N. Vaidya, "A spatial backoff algorithm using the joint control of carrier sense threshold and transmission rate," in Sensor, Mesh and Ad Hoc Communications and Networks, 2007. SECON'07. 4th Annual IEEE Communications Society Conference on. IEEE, 2007, pp. 501-511.

[12] Z. Chen, X. Yang, and N. Vaidya, "Dynamic spatial backoff in fading environments," in Mobile Ad Hoc and Sensor Systems, 2008. MASS 2008. 5th IEEE International Conference on. IEEE, 2008, pp. 255264.

[13] T.-S. Kim, Y. Yang, and J. C. Hou, "Modeling of ieee 802.11 based multi-rate wireless ad hoc networks and its application towards optimal network operation,” 2007. [Online]. Available: http://hdl.handle.net/2142/11326

[14] T.-S. Kim, H. Lim, and J. C. Hou, "Understanding and improving the spatial reuse in multihop wireless networks," Mobile Computing, IEEE Transactions on, vol. 7, no. 10, pp. 1200-1212, 2008. 